\title{
Lengua y etnicidad: una relación con matices
}

\author{
Masatsugu Matsuo
}

\begin{abstract}
Language is one of the main features that allow us to differentiate one ethnic group from another. However, the relationship of ethnic groups towards their languages is not invariable and the examination of particular cases reflects a wide array of nuances. Given this ambivalence of the subject, this paper seeks to examine which properties can be assumed as necessary in order to establish a link between a language and an ethnic group. By referring to some case studies, the author demonstrates how the link between language and group's identity has been maintained sometimes more strongly by a shared perception than by an effective use of the language in question.
\end{abstract}

\section{Introducción}

Desde finales del siglo veinte la prevalencia de los conflictos étnicos ha llamado en gran medida la atención académica. Sin embargo, recientemente un importante número de profesionales ha argumentado que los conflictos étnicos no son causados directamente por la diferencia étnica o la pluralidad (multietnicidad) dentro de un Estado, ${ }^{1}$ aunque no podemos dejar de lado que hay muchos argumentos que favorecen la idea contraria. ${ }^{2}$ Pero a pesar de la presunta poca relevancia de la etnicidad como un factor que provoca el mal llamado conflicto étnico, hay una pregunta importante que aún está sin resolver: ¿por qué tantos conflictos internos violentos resultan ser conflictos étnicos y, más precisamente, por qué tantos conflictos internos se luchan desde el frente étnico o a lo largo

1 Ver argumentos de Fearon y Laitin, 1996: 716-717; Henderson y Singer, 2000: 293; Holsti, 1998: 109-110, y Lake y Rothschild, 1998: 4, 7.

2 Aunque no es este el lugar para detallarlos, no se puede dejar de anotar que existen muchos argumentos intermedios. Por ejemplo, la polarización en dos grupos o el despojo de un grupo mayoritario pueden aumentar el riesgo de conflicto interno, mientras la racionalización misma no lo hace. (Collier y Hoeffler, 2000: 26, 2003 : 537-538). 
de las fronteras étnicas? En este documento exploraremos una propiedad de los "grupos étnicos" como un paso hacia la respuesta final a esta pregunta.

Aunque la lengua no es el único o más importante factor que distingue a los grupos étnicos, muchos profesionales han argumentado hasta ahora que ésta se ubica entre los factores claves que permiten diferenciar a un grupo étnico de otros. ${ }^{3}$ Muchos autores admiten que, en casos extremos, la lengua es considerada como el único símbolo que representa la relación completa de los grupos étnicos que se encuentran en conflicto (Nelde, 1987: 35; Ozolins, 1996: 182). Pero estos autores rara vez han ido más allá de esta afirmación categórica, como si la relación entre una lengua particular y un grupo étnico-nacional fuera algo que se pudiera dar por sentado. El presente artículo constituye un intento de ir un poco más lejos y explorar las modalidades de las relaciones entre una lengua y un grupo étnico, ya que, como una vez Uldis Ozolins insinuó, hay "una variedad de posibles relaciones entre una lengua y la etnicidad, en diferentes contextos históricos" (Ozolins, 1996: 182). Nuestro objetivo es dar una respuesta a por qué esto se presenta así.

En la siguiente sección, prepararemos el terreno en el que trataremos este asunto, exponiendo las premisas y las suposiciones relacionadas. En las secciones 3 y 4 haremos un breve análisis teórico de una propiedad que funciona como marcador límite de los grupos étnicos y plantearemos dos condiciones (la homogeneidad interna y la heterogeneidad externa) con las que el marcador de límites debe cumplir. En la sección 5, con el análisis de la naturaleza de la relación ente dos o más lenguas, mostraremos que la relación real de las lenguas es tal que se puede hacer una identificación y distinción de las lenguas en cuestión. En otras palabras, argumentaremos que lo que importa es la percepción o interpretación y no la realidad en cuanto a la identificación y diferenciación de las lenguas. En la sexta sección citaremos unos pocos casos que muestran la relevancia de la percepción en la identificación y diferenciación de una lengua. En la última sección concluiremos el ensayo planteando que es la percepción y no la realidad la que importa, y que esto es lo que ocasiona que la asociación de una lengua dada con un grupo étnico-nacional sea muy flexible.

\section{Enfoque de la relación entre lengua y etnicidad}

Como es sabido, tanto la lengua como la etnicidad (grupo étnico o étniconacional) son conceptos muy difíciles. Muchos volúmenes de libros serían necesarios para la definición de estos términos. Por lo tanto, como primera aproximación, nos vemos obligados a empezar preparando el terreno de nuestra discusión.

3 Para un ejemplo, ver Das Gupta, 1975: 470. 
En primer lugar, tenemos que resolver, o al menos aclarar, la cuestión de la terminología como una de nuestras variables, dado que existen muchos (tal vez demasiados) y muy confusos términos para la etnicidad o grupo étnico. Por ejemplo, además del término "étnico" nos encontramos con términos como "comunal", "nacional", "nacionalidad", "mini-nacional", "sub-nacional", "étnico-nacional", étnico-político", "étnico-regional", etc. En relación con el grupo étnico o la etnicidad, el término "nación" es usado muy frecuentemente en un sentido muy parecido, aunque algunos autores lo diferencien de manera estricta. En este artículo usaremos el término "grupo étnico-nacional" o "etnicidad" en vez de otros términos para abarcar el concepto de "nación" y "grupo étnico", o para poder explorar el asunto de las dos formas, es decir, tanto a nivel estatal como sub-estatal. Ahora bien, aun cuando una "nación" y un grupo étnico se puedan, o se deban, distinguir conceptualmente, la mayoría de los argumentos en este artículo, si no todos, aplican a los dos. Además, no utilizaremos sub-categorías o sub-divisiones como "nacionalidad", "minoría étnica", "pueblos indígenas", "tribus" y demás. Aunque estos y otros términos más pueden ser de utilidad en ciertas circunstancias, nos quedaremos con "grupo étnico-nacional".

En segundo lugar, tenemos que limitar nuestra atención más estrechamente a cierta faceta de la relación entre la lengua y un grupo étnico-nacional. En este artículo nos centraremos en un aspecto de esta relación, es decir, nos preguntaremos si la lengua define un grupo étnico-nacional y cómo lo hace. Para decirlo de otra manera, nuestro enfoque radica en la lengua, vista como un marcador de fronteras de un grupo étnico-nacional. Este enfoque tan exacto deja muchos temas sin tocar, de los cuales indicaremos dos. La primera omisión cubre aquellos casos en los que un grupo étnico-nacional parece definir la lengua, pero no sucede lo contrario. De acuerdo con Earnest Gellner, el Estado moderno ha creado una comunidad de lenguaje caracterizada por una lengua escrita común o de "alta cultura" (Gellner, 1983: 33-34). Como William Safran señala, las relaciones caracterizadas entre la lengua y un grupo étnico son bidireccionales (Safran, 1999: 92). La lengua y la etnicidad se constituyen la una a la otra. Sin embargo, en este ensayo nos enfocaremos sobre la lengua como factor distintivo de un grupo étnico-nacional. El segundo tema que no se abordará, y cuya omisión sin duda es seria, versa sobre cómo la identidad y el interés forman dos dimensiones importantes en la relación lengua-etnicidad. Nuestro enfoque en la lengua como característica de etnicidad deja a esta última por fuera, a pesar de su creciente importancia (Glazer y Moynihan, 1975: 7, 12-13; Inglehart y Woodward, 1967: 32). 
En tercer lugar, cuando tratamos con la relación entre variables, lengua y grupo étnico-nacional, es conveniente teóricamente, y tal vez más gratificante, mantener una variable constante. Por eso, asumiremos en nuestra discusión que un grupo étnico-nacional está dado y es fijo, excepto en casos en los que la lengua parezca ser la causa del cambio en un grupo étnico. Esto no significa, sin embargo, que creamos en la fijeza primordial de un grupo.

En cuarto lugar, se argumenta, por lo general, que para constituir un grupo étnico-nacional, o cualquier grupo humano, la colectividad humana debe compartir algunas propiedades objetivas y un sentido de pertenencia a la comunidad. La gente comparte algunas propiedades como la lengua o las costumbres, sin por ello compartir el sentido de pertenencia. Pero esta gente no constituye un grupo étnico-nacional sino comparte un sentido de pertenencia. Aun desde nuestra perspectiva limitada de la lengua como marcador de una etnicidad, por consiguiente, como Anthony Smith advierte (Smith, 1986: 211), debemos manejar los dos aspectos: el aspecto objetivo y el aspecto subjetivo de este sentido de pertenencia. Pero aquí no vamos a discutir el sentido de pertenencia, sino que vamos solamente a tratar la lengua como "definidora" de un grupo étniconacional. No obstante, esta propiedad objetiva, aparentemente, involucra una gran cantidad de dimensiones subjetivas, como veremos más adelante.

\section{Propiedades objetivas compartidas}

Usualmente se afirma que un grupo étnico-nacional es un grupo de personas que comparten algunas propiedades objetivas. Las propiedades objetivas o atribuidas incluyen aquí ancestros, historia, territorio (lugar de habitación ancestral), vida económica, lengua, religión, cultura, patrones de comportamiento, características físicas (como el color de la piel), etc. Estas características son ampliamente dadas a un individuo y no pueden ser cambiadas fácilmente por él y, por lo general, se heredan a través de las generaciones. Si estas propiedades son para definir un grupo étnico-nacional, es decir, para distinguirlo de otro, deberían ser compartidas por sus miembros y heredadas de una generación a otra. La mayoría de estas propiedades son visibles y objetivas. Ellas dan un fundamento objetivo a la existencia de un grupo étnico-nacional. Es decir, un grupo étnico-nacional es una colectividad compartiendo estas propiedades.

Hay una pregunta muy interesante respecto al hecho de compartir las propiedades. ¿Debería un grupo étnico-nacional compartir todas o casi todas las propiedades o atributos antes mencionados, o cuántos de ellos debería compartir? Ya que nos enfocamos en la lengua, sencillamente simplificaremos la pregunta: ¿debería un grupo étnico-nacional compartir la lengua o no? En las próximas secciones trataremos de responder esta pregunta. 
Por otra parte, el hecho de que dos o más grupos compartan una o más propiedades no garantiza que ellos pertenezcan a, o constituyan, un solo grupo. Las características objetivas compartidas son la materia prima de los criterios para definir a un grupo étnico, en lugar de los criterios en sí (Rothchild, 1981: 95). Esto también es cierto para la lengua. Compartir la misma lengua no garantiza que los "usuarios" pertenezcan al mismo grupo étnico-nacional (Das Gupta, 1975: 470; Safran, 1999: 81). De esto podemos concluir, que las propiedades objetivas, compartidas por un grupo étnico-nacional deben ser aquellas que puedan ser utilizadas para distinguirlo de otros grupos. Llamemos a estas propiedades "distintivas". Las propiedades distintivas son las que funcionan como marcadores de límites entre los grupos étnico-nacionales. Para saber cuál de estas propiedades juega un rol de rasgo distintivo, tendremos en cuenta las condiciones históricas del grupo étnico-nacional en cuestión.

El conflicto de dos grandes grupos étnico-nacionales en Bélgica es bien conocido. Como los dos grupos son católicos y comparten la religión, la lengua (holandés y francés) y el territorio o la región (norte y sur), estas se convirtieron en las propiedades distintivas que distinguían a los dos grupos. De la misma manera, en la India independiente, la partición privó a la religión de la función de "marcador" de etnicidad y llevó a la lengua a ser un marcador de ésta, conduciendo rápidamente a la creación de estados lingüísticos, empezando con el caso de Andhra Pradesh (Inglehart and Woodward, 1967: 32). Por el contrario, la religión y el denominador religioso o de secta son propiedades distintivas en Irlanda del Norte y el Líbano. Para resumir, lo que importa al distinguir los grupos étnicos son las propiedades distintivas y, como marcador de límite de etnicidad, la lengua debe ser una propiedad distintiva separada o combinada con otras propiedades.

Aquí debemos hacer una aclaración en el ámbito de la distinción o la propiedad distintiva. Una propiedad distintiva o un grupo de propiedades distintivas no necesitan "distinguir" un grupo étnico-nacional de los demás grupos étnicos nacionales. Esto se puede entender fácilmente cuando consideramos situaciones en las que la pertenencia importa. Algunos investigadores plantean que un grupo étnico-nacional llega a validar su nombre solamente cuando está en contacto con otros grupos. En otras palabras, un grupo étnico-nacional llega a ser verdaderamente un grupo étnico cuando, y solamente cuando, el grupo necesita distinguirse de otros. Por lo tanto, un rasgo distintivo o un conjunto de ellos sólo tiene que distinguir al grupo en cuestión de los que están en contacto con él de alguna u otra manera. De este modo, si un grupo étnico-nacional no está en contacto con otro grupo, no tiene necesidad de distinguirse de los demás (al menos en teoría), es decir, no tiene necesidad de tener un nombre para 
lograr una diferenciación. Vemos esto ejemplificado en el hecho de que pueblos indígenas del mundo como los ainu en Japón, los maoris en Nueva Zelanda y muchas minorías étnicas en la región Evenki de Siberia han utilizado nombres para designarse a sí mismos que significan sencillamente "ser humano". Una propiedad distintiva, o un grupo de ellas, solamente tiene que distinguir al grupo étnico-nacional de un cierto grupo o grupos, pero no de todos los demás. Las propiedades objetivas se necesitan para ser distinto pero no para ser un grupo único, es decir, que sean propias sólo de un grupo étnico-nacional o lo distingan todos los demás. El idioma francés es una propiedad distintiva de los franco-canadienses o quebequenses que los distingue de los anglo-parlantes (la gran mayoría) en Canadá, pero el francés no los distingue de los franceses en Francia, porque no hay necesidad de que los quebequenses se distingan de los franceses europeos. Si fuera necesario diferenciarlos por la lengua habría que recurrir al dialecto “juoal” del Quebec y al francés estándar de Francia (Weinstein, 1989: 54, 57-58).

En resumen, una propiedad distintiva debe ser vista como una propiedad que puede distinguir al grupo étnico-nacional de otro específico, pero no de todos los demás grupos. Es cierto, sin embargo, que en muchos casos una propiedad distintiva, o un grupo de ellas, tiende a percibirse como única para el grupo en cuestión, es decir, como una propiedad que los separa de los demás. Desde esta perspectiva, es muy normal que para consagrar la unicidad del grupo algunos grupos deban intentar establecer su unicidad en su nombre, aun cuando no tengan ninguna propiedad particular o única en absoluto. De hecho, esto es muy común, como en el caso de llamar indonesio o malasio al lenguaje malay, lo cual es un ejemplo típico de este fenómeno.

\section{La homogenidad interna y la heterogenidad externa: condiciones para una propiedad distintiva}

Hasta ahora hemos usado el término "compartir" sin ninguna definición. En esta sección examinaremos el significado de "compartir", haciendo especial énfasis en la lengua.

Supongamos que $\mathrm{X}$ representa una propiedad distintiva que diferencia un grupo étnico-nacional de otros. Para que sea una propiedad distintiva, $\mathrm{X}$ debe cumplir con las siguientes dos condiciones:

1. X es compartida con un número suficiente de miembros del grupo étnico-nacional (homogeneidad o proposición de similaridad). 
2. X no aplica a (y no es compartida por) la mayoría de los miembros de otro(s) grupo(s) étnico-nacional(es) (heterogeneidad o proposición de diferencia).

La primera proposición requiere que el grupo étnico sea homogéneo internamente en lo que respecta a X. La segunda requiere que los dos grupos, al ser comparados, sean heterogéneos entre sí en lo que respecta a X. Combinadas, las proposiciones requieren homogeneidad interna y heterogeneidad externa de X. En otras palabras, X debe ser compartida por los miembros del grupo en cuestión pero no por los otros. Cuando analizamos la lengua desde la perspectiva de un marcador de límite étnico-nacional, puede parecer al principio que sólo tenemos que reemplazar a X por la lengua. Pero entonces surge una pregunta difícil: ¿debería la lengua, que supuestamente diferencia a un grupo étnico-nacional de otro, ser hablada y escrita por la mayoría de los miembros del grupo, o es suficiente que sea hablada o entendida? Retomaremos este aspecto con más detalle después.

La relevancia de las dos condiciones se puede observar en conflictos en tornoa la lengua históricos y contemporáneos, especialmente en los que llamo "conflictos arraigados". En un conflicto arraigado un grupo étnico-nacional busca diferenciarse a sí mismo de otros en términos de la lengua utilizada e intenta homogeneizarla, como en la Hungría del siglo XIX y en la Moldavia post-soviética (Matsuo, 1999:96-99). ${ }^{4}$ Se puede decir que, en este caso como en otros, las dos condiciones se convierten en una especie de norma.

Las dos condiciones no son sólo políticamente importantes, sino también una consideración teórica. Cuando estas condiciones, la homogeneidad interna y la heterogeneidad externa, no se cumplen, muchos estudios previos sobre etnicidad han llegado incorrectamente a una u otra de las siguientes conclusiones. Por una parte, el grupo de las propiedades distintivas, o la propiedad distintiva, podría terminar no siendo la característica de un grupo étnico-nacional, porque no era(n) compartida(s) por los miembros, o era(n) compartida(s) por los miembros de otros grupos. Por otra parte, se podría concluir que el "grupo étnico-nacional" no era en efecto un grupo distinto y separado de otro, porque el grupo no cumplía las condiciones necesarias de las propiedades distintivas. En este último caso, la propiedad distintiva $\mathrm{X}$ es tratada como un criterio absoluto en el sentido de que cada grupo debe compartirla. Las dos conclusiones son erradas en la mayoría de los casos. El error más serio en estos argumentos es que han caído en el sofisma de pensar solamente en la realidad y han ignorado totalmente el asunto de la percepción. Cuando consideramos la relación de una

4 En otros muchos casos, ni la homogenización o el esfuerzo de diferenciación son predominantes. Para ejemplos ver Matsuo, 1999: 92-97. 
propiedad distintiva, especialmente la lengua, con un grupo étnico-nacional, debemos considerarlas ambas, la realidad y la percepción. La última perspectiva, es decir, la perspectiva de la percepción, es en realidad necesaria.

Aunque después, analizaremos el asunto más detalladamente con referencia a la lengua, consideremos, como una ilustración de la importancia de la percepción, el problema de ascendencia o del origen de un grupo étnico-nacional. La ascendencia común se considera como una de las bases objetivas del grupo, si nos remontamos a la historia. Sin embargo, es frecuente el caso de que la genealogía de un grupo étnico-nacional desaparezca en algún lugar del oscuro pasado de la historia, al menos en el sentido científico estricto de la palabra. Además es muy difícil encontrar un grupo étnico-nacional "puro" sin alguna mezcla con otros grupos. En este sentido, si nos quedamos con la ascendencia común como base o propiedad distintiva de un grupo, no existiría objetivamente tal cosa como un grupo étnico-nacional, en un sentido estricto. Pero en lo que concierne a la etnicidad, el concepto de ascendencia no es equivocado, sino la negación del estatus de grupo étnico-nacional sobre la base de no tener un origen común en el sentido histórico (objetivo). En otras palabras, el error no está en el hecho de que no exista un grupo que comparta la genealogía en un sentido estricto, el error está en la premisa de que un grupo debería tener una sola ascendencia como un hecho histórico objetivo. Como Walker Connor señala exhaustivamente, un grupo étnico es el grupo humano más grandioso caracterizado por compartir el mito de la ascendencia común. Pero importa poco si el mito corresponde a un hecho histórico (Connor, 1987: 211).

Generalizando, lo errado es pensar que las propiedades distintivas son hechos objetivos (históricos) y realidades. Para ponerlo de manera diferente, lo que importa es que los miembros de un grupo en cuestión (y los no-miembros también) crean en los ancestros comunes o no (Connor, 1987: 205-206). Con certeza, es claramente una exageración decir que es primordial que haya una característica objetiva y no visible para corroborar la existencia de un grupo étnico-nacional (Connor, 1987: 202). Un grupo étnico-nacional no necesita en realidad compartir sus ancestros y de hecho, por lo general, no necesita compartir aspectos distintivos. La condición suficiente es que la propiedad distintiva, la genealogía, en este caso, sea percibida o crea ser compartida con los otros miembros del grupo. Por lo tanto, lo que importa no es lo que realmente se comparte sino lo que se cree o se percibe como compartido. Esto es relevante especialmente con respecto a la lengua, como veremos más adelante.

A partir estas observaciones podemos decir en esta etapa de la investigación que un conjunto de propiedades distintivas que diferencia a un grupo étnico-nacional de otro no necesita necesariamente ser compartido, sino que 
los miembros del grupo deben creer o percibir que tal conjunto se comparte con los otros miembros. Esto no significa, por supuesto, que los rasgos distintivos no puedan o no deban ser compartidos. Esta percepción de intercambio de propiedades puede funcionar como evidencia de la existencia de un grupo étnico-nacional, puede influenciar los comportamientos de sus miembros y puede tener un efecto político importante. Por lo tanto, las propiedades compartidas percibidas, como la genealogía, pueden funcionar como un mito.

\section{La relación de la lengua: realidad y percepción}

En las secciones anteriores hemos expuesto dos condiciones que se deben cumplir cuando la lengua (o cualquier otra propiedad) diferencia a un grupo étnico-nacional de otro. El idioma debe ser compartido por los miembros del grupo pero no con los miembros de otros grupos. Las condiciones comprenden tres grandes componentes, o un predicado y dos argumentos: compartir la lengua, un grupo étnico-nacional, una lengua específica. Para aclarar el rol de la lengua como un marcador de grupo étnico-nacional, esta debe ser identificada y separada de otro grupo, y la lengua que lo diferencia debe ser separada de otras lenguas. Como dijimos anteriormente, asumimos como dada la identificación de un grupo, por lo tanto no analizaremos cómo se identifica la lengua.

Para que una lengua funcione como un marcador de límites de etnicidad, ésta debe ser diferenciada de otras lenguas. Es, sin embargo, una tarea muy difícil aun para los lingüistas decidir si dos lenguas relacionadas (o variedades de lenguas) son la misma o dos totalmente diferentes. Según argumenta Bernard Comrie:

Es difícil o imposible, en muchos casos, decidir si dos variedades de discursos deban ser considerados lenguas diferentes o sencillamente dialectos diferentes de la misma lengua $[\ldots]$ pero estas decisiones han sido frecuentemente más en el campo político o social que en el campo estrictamente lingüístico (Comrie, 1990: 2). ${ }^{5}$

Yo intenté una vez explicar (Matsuo, 2005) por qué esta tarea tan difícil de diferenciación (o identificación) de lenguas no sólo es posible sino que también ha sido realizada "en el campo político y social".

5 Por consiguiente, como dice un autor : "Desde una perspectiva estrictamente lingüística, [...] una lengua es un dialecto que tiene un ejército, una armada y una fuerza aérea, esa es la única diferencia real desde el punto de vista lingüístico" (Gianrenzo Clivio, citado en :Steinberg, 1987: 199). 
La relación de dos lenguas (o dialectos) puede ser representada esquemáticamente como lo muestra la figura 1.

\section{Figura 1: Relación básica}

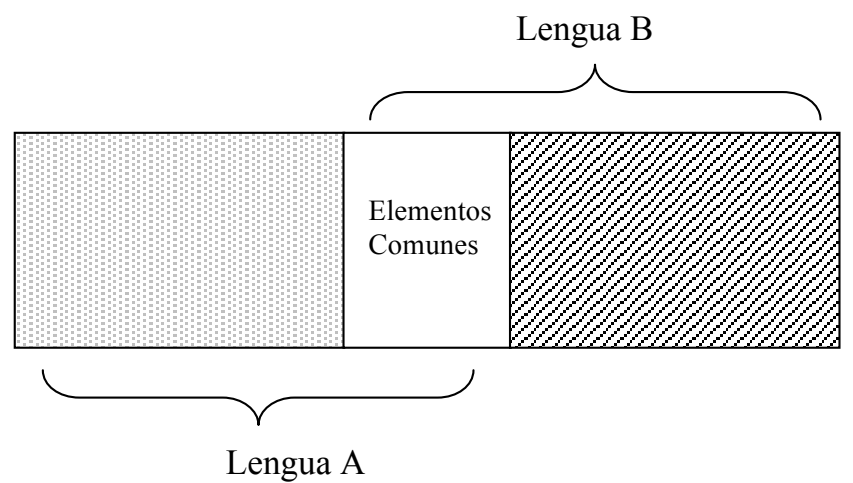

Por una parte, las dos lenguas en cuestión difieren una de la otra en reglas gramaticales (sintaxis), en elementos léxicos (vocabulario), o en el sistema de sonidos (pronunciación), o en alguna combinación de todas ellas. Por otra parte, sin embargo, siempre hay elementos comunes (aun si excluimos el préstamo de palabras) que se comparten. Aunque no es muy precisa, la descripción sería suficiente desde el punto de vista puramente lingüístico. En realidad, más de dos lenguas (o variedades de lenguas) están a menudo involucradas, como en el caso del dialecto continuo y del creole continuo (Comrie, 1990: 3, 17; Friedman, 1993: 160; Kalogjera, 1985: 94). Aun para un idioma nacional es posible decir que, en el caso de las lenguas nórdicas, el "noruego es danés hablado en Suecia" (Haugen, 1990: 151).

Pero, al contrario de los lingüistas que afirman que "la escritura no es una lengua, sino sencillamente un modo de grabar el mensaje con un medio de marcas visibles", el sistema de escritura y el grupo de letras (alfabeto) deberían ser considerados como un componente esencial de la lengua, como han concluido varios investigadores (Calvet, 1998: 153; Schieffelin and Doucet, 1998: 285). El hecho es que han existido muchos conflictos debido al sistema de escritura. Para mencionar unos pocos casos puntuales, ha habido conflictos en Galicia bajo el poder polaco a mediados del siglo XIX (Subtelny, 1988: 513-514), Pakistán oriental (ahora Bangladesh) antes de la independencia (Musa, 1996: 75-76), la República Soviética de Moldavia (Eyal, 1990: 126-127), y en la Unión Soviética debido a la latinización de la escritura para los idiomas turcos (Lazzerini, 1985: 116-117). Además, si no lleva directamente a la violencia, el sistema de escritu- 
ra ha sido empleado para distinguir grupos étnico-nacionales, uno a uno o en combinación con todos los elementos. El serbio y el croata estandarizado de la antigua Yugoslavia eran distinguidos por el alfabeto cirílico y el latino (Corbett, 1990: 128), el hindi y el punjabi fueron distinguidos por los alfabetos devanagari y grumukhi (Dale, 1980: 10; Dua, 1996). ${ }^{6}$

Se supone que la relación entre dos lenguas (o variedades de lengua como dialectos) descrita por la figura 1 es fiel reflejo de la realidad. De esta única realidad, como fue mostrado en Matsuo (2005), son posibles una variedad de interpretaciones. La relación "real" de la lengua es la que nos permite diferentes interpretaciones pues, como en cualquier otro fenómeno social, un hecho permite muchas interpretaciones diferentes. Por lo tanto, mientras algunos pueden identificar dos lenguas como una sola, viendo los dialectos como parte de una lengua, otros pueden diferenciarlos, viéndolos como lenguas diferentes. Aunque hay muchos ejemplos que pueden ser citados para los dos casos, ${ }^{7}$ nos limitaremos a mencionar uno o dos de cada uno de ellos.

En primer lugar, pese a las grandes diferencias geográficas, el chino y el árabe son considerados como una sola lengua. El inglés puede ser otro ejemplo. Este tipo de identificación no está solamente limitada a las "grandes" lenguas habladas en áreas extensas y por mucha gente. Por ejemplo, los moldavianos han sido considerados como un pueblo de una cabeza y dos bocas porque tienen dos dialectos, mutua y cercanamente incomprensibles, el erzia y el moksha (Kreindler ,1985: 237-238). En el siglo XIX, las autoridades rusas consideraban al ucraniano (o pequeño ruso) como un dialecto ruso aun cuando estaba contaminado por la influencia polaca, mientras que los nacionalistas ucranianos declaraban tener una lengua separada (Solchanyk, 1985: 58). Y el bielorruso fue considerado muchas veces como un dialecto polaco, ruso o ucraniano (Wexler, 1985: 38). La percepción de un gallego en la península ibérica nos ofrece otro ejemplo (Parkinson, 1990: 250).

También encontramos muchos casos de diferenciación en las percepciones, una gran semejanza (o entendimiento mutuo) no necesariamente previene diferenciaciones entre dos lenguas (dos variedades de lengua). Algunos argumentan que el escocés (también llamado inglés escocés y, como su nombre lo dice, percibido como un dialecto geográfico del inglés) es una lengua distinta del inglés (Aitkin, 1990: 76). Tal vez la India ha sido una especie de laboratorio para este tipo de percepciones, porque la identificación de una lengua distinta ha sido inseparablemente conectada con su autonomía étnico-nacional en forma de "Estado lingüístico" (Chaklader, 1990: 130-131, 207-208).

6 Aunque en el caso extremo de lenguas no relacionadas genéticamente, éstas comparten las propiedades comunes de todas las lenguas humanas. Véase: Chomsky, 1975: 29.

7 Para ejemplos, ver Matsuo (2005), especialmente pp. 192-202. 


\section{Percepción compartida de una lengua}

En las secciones anteriores hemos mostrado que las lenguas (o variedades como dialectos) pueden ser identificadas o diferenciadas en una amplia percepción, independientemente de su cercanía real o distancia. En esta sección analizaremos el predicado de nuestras dos condiciones, es decir, el hecho de compartir la lengua por parte de los miembros del grupo étnico-nacional.

Es teóricamente posible encontrar el alcance que los miembros de un grupo étnico-nacional le han dado a la lengua (en cualquier forma que se defina "compartir"). Y de hecho, se han realizado muchos intentos en forma de censo, conteniendo temas de la lengua materna, la primera lengua, etc. En este caso, nuevamente, no es la realidad sino la percepción la que importa. En otros casos, al menos, una lengua no necesita ser hablada o entendida por la mayoría de un grupo étnico-nacional dado. Por ejemplo, el reino de Hungría estuvo bajo el mandato de los Habsburgo en el siglo XVIII y en 1784 el emperador José II declaró el alemán como la lengua oficial de la administración y educación en todo el imperio (Fichtner, 1997: 24). La declaración de “alemanización” causó una violenta reacción por parte de los húngaros, especialmente de la nobleza (Barany, 1971: 262-263). Contrariando la política imperial de "alemanización", la nobleza adoptó el magiar (húngaro) como su lengua común, aunque la lengua húngara parecía obsoleta entre la nobleza (Inglehart and Woodward, 1967: 34). Como ejemplifica este caso, la lengua compartida no es un objetivo necesario, sino una condición intersubjetiva. En otras palabras, es suficiente que una lengua (el húngaro en este caso) sea percibida (creída o imaginada) para ser compartida por sus miembros. En este sentido, un grupo étnico-nacional, podría ser una comunidad imaginada basada en la lengua (Anderson, 1983: 15).

El caso irlandés es tal vez el ejemplo más claro de la importancia de la percepción de una lengua compartida. Miremos las estadísticas de la lengua irlandesa. Los hablantes gaélicos (irlandeses) eran menos del 20\% de la población antes de la independencia en 1922 (Hindley, 1990: 15, 23). No puede entonces decirse que la lengua es realmente compartida por sus miembros. Sin embargo, la lengua gaélica es un rasgo característico de los pueblos irlandeses en percepción y en sentido simbólico. El gaélico es percibido como la lengua de los irlandeses. Es por esta razón que el gobierno irlandés declaró al gaélico como la lengua nacional, esforzándose en su promoción y enseñándola en las escuelas. En el movimiento de independencia de finales del siglo XIX y principios del siglo XX, también el gaélico fue la lengua símbolo del movimiento. Por consiguiente, la liga gaélica, que buscaba reavivar y promocionar dicha lengua, jugó un papel decisivo en la lucha por la independencia. En este caso, la lengua gaélica no era realmente compartida por la mayoría, pero tuvo un importante rol 
durante la independencia como símbolo de grupo. El caso de Irlanda muestra típicamente que lo que importa no es la realidad del compartir, sino la percepción (o el imaginario) de compartir. En resumidas cuentas, una lengua no necesita ser compartida realmente por la mayoría de los miembros de un grupo étniconacional. Es suficiente, en algunos casos al menos, que sea percibida, creída o fomentada por sus miembros.

\section{Conclusión}

En este ensayo hemos propuesto inicialmente dos condiciones que deben ser cumplidas cuando el lenguaje funciona como un marcador étnico-nacional. Posteriormente, hemos mostrado que el cumplimiento de las condiciones no depende de la realidad sino que depende doblemente de la percepción. Por un lado, las lenguas pueden ser libremente identificadas o diferenciadas, sin la distinción de la percepción o de la similitud. Por otra parte, el compartir una lengua entre los miembros de un grupo a veces es un asunto de percepción más que de la realidad. Por consiguiente, la asociación de una lengua con un grupo étnico-nacional (aunque sea constante como lo hicimos) puede ser muy flexible, y por eso la lengua proporciona un criterio flexible y conveniente por medio del cual un grupo étnico cultural puede ser identificado.

En este artículo no nos hemos ocupado de la maleabilidad de la identificación étnico-nacional, aunque sí tomamos en cuenta su variación, reconociendo que la posibilidad de diferentes identificaciones étnico-nacionales puede expandirse ampliamente. Tampoco hemos considerado políticas o movimientos en torno a las lenguas que intentan ajustar la realidad a la percepción, imponiendo, por ejemplo, una lengua dominante o mayoritaria a los grupos minoritarios. $\mathrm{Si}$ tomamos en cuenta tal intento de confirmación de dos condiciones, se abre la posibilidad de relacionar una lengua con un grupo étnico-nacional. Estos temas entrarán en consideración en la agenda para nuestra próxima investigación.

\section{Bibliografía}

-Aitkin, Adam J. (1990), "The good old scots tongue: does scots have an identity?”, en: Einar Haugen, et al (eds.), Minority languages today, Edinburgh, Edinburgh University Press, pp. 72-90.

-Anderson, Benedict (1983), Imagined communities: reflections on the origin and spread of nationalism, London, Verso. 
-Barany, George (1971), "Hungary: from aristocratic to proletarian nationalism", en: Sugar, Peter F. e Ivo John Lederer (eds.), Nationalism in Eastern Europe, Seattle, University of Washington Press, pp. 259-309.

-Bloomfield, Leonard (1969, 1933), Language, London, George Allen and Unwin.

-Calvet, Louis-Jean (1998), Language wars and linguistic politics, Oxford, Oxford University Press.

-Chaklader, Snehamoy (1990), Sociolinguistics: a guide to language problems in india, New Delhi, Mittal

-Chomsky, Noam (1975), Reflections on language, New York: Pantheon Books.

-Collier, Paul and Anke Hoeffler (2000), Greed and grievance in civil war, world bank policy research working paper 2355, World Bank (http:/ /www.worldbank.org/ research/PDF)

-Collier, Paul y Anke Hoeffler (2003), "On economic causes of civil war", en: Sandler; Todd y Keith Hartley (eds.), The economics of conflict vol. 1 theory, cheltenham: Edward Elgar, pp. 529-539. Reprinted from Oxford Economic Papers, 50(4) (1998), 563-73

-Comrie, Bernard (ed.) (1990a), The Major languages of western europe, London, Routledge.

-Comrie, Bernard (ed.) (1990b), The major languages of eastern europe, London, Routledge.

-Connor, Walker (1987), "Ethnonationalism”, en: Weiner, Myron and Samuel P. Huntington (eds.), Understanding political development: an analytic study, Boston, Little, Brown and Company.

-Corbett, Greville (1990), "Serbo-Croat,” en: Comrie, Bernard (ed.) (1990b), The Major Languages of Eastern Europe, London: Routledge. 
-Dale, Ian R. H. (1980), "Digraphia", en: International Journal of the Sociology of Language, 26, pp. 5-13

-Das Gupta, Jyotirindra (1975), "Ethnicity, Language Demands and National Development in India", en: Glazer, Nathan and Daniel P. Moynihan (eds.) (1975), Ethnicity: Theory and Experience, Cambridge, MA: Harvard University Press.

-Dua, Hans Raj (1996), “The Politics of Language Conflict: Implications for Language Planning and Political Theory”, en: Language Problems and Language Planning, 20(1), pp. 1-17.

-Eyal, Jonathan (1990), "Moldavians", en: Graham Smith (ed.) (1990), The Nationalities Question in the Soviet Union, London: Longman, pp. 123-141

-Fearon, James D. and David D. Laitin (1996), "Explaining Interethnic Cooperation", en: American Political Science Review, 90(4), pp. 715-735

-Fichtner, Paula Sutter (1997), The Habsburg Empire: From Dynasticism to Multinationalism, Malabar, FL: Krieger.

-Fishman, Joshua A. (ed.) (1999), Handbook of Language and Ethnic Identity, New York: Oxford University Press.

-Fishman, Joshua A. (1999), "Concluding Comments", en: Fishman, Joshua A. (ed.), Handbook of Language and Ethnic Identity, New York: Oxford University Press, pp. 444-454

-Friedman, Victor A. (1993), "The First Philological Conference for the Establishment of the Macedonian Alphabet and Macedonian Literary Language: Its Precedents and Consequences", en: Fishman, Joshua A. (ed.) (1993), The Earliest Stage of Language Planning: The "First Congress" Phenomenon, Berlin: Mouton de Gruyter, pp. 159-180

-Gellner, Ernest (1983), Nations and Nationalism, Oxford: Basil Blackwell. 
-Glazer, Nathan and Daniel P. Moynihan (1975), "Introduction," en: Glazer and Moynihan (eds.) (1975), Ethnicity: theory and experience, Cambridge, MA, Harvard University Press.

-Glazer, Nathan and Daniel P. Moynihan (eds.) (1975), Ethnicity: theory and experience, Cambridge, MA, Harvard University Press.

-Haugen, Einar (1990), "Danish, norwegian and swedish" en: Comrie, Bernard (ed.) (1990a), The major languages of western europe, London, Routledge, pp. 147-169.

-Henderson, Errol A. and John David Singer (2000), "Civil war in the postcolonial world, 1946-92”, en: Journal of Peace Research, 38(3), pp. 275-299.

-Hindley, Reg. (1990), The death of the irish language: a qualified obituary, London, Routledge

-Holsti, Kalevi J. (1998), "International relations theory and domestic war in the third world: the limits of relevance", en: Neuman, Stephanie G. (ed.), International relations theory and the third world, Houndmills and London, Macmillan, 103-132.

-Inglehart, Ronald F. and Margarett Woodward (1967) "Language conflicts and political community", en: Comparative studies in society and history, 10(1), pp. 27-45.

-Kalogjera, Damir (1985), "Attitudes toward serbo-croatian language varieties", en: International journal of the sociology of language, 52, 93-109.

-Kreindler, Isabelle T. (1985), "The mordvinian langauges: a survival saga", en: Kreindler, Isabelle T. (ed.) (1985), Sociolinguistic perspectives on soviet national languages: their past, present and future, Berlin, Mouton de Gruyter pp. 237-264.

-Kreindler, Isabelle T. (ed.) (1985), Sociolinguistic perspectives on soviet national languages: their past, present and future, Berlin Mouton de Gruyter.

-Lake, David A and Donald Rothschild (1998), "Spreading fear: the genesis of transnational ethnic conflict", en: Lake, David A and Donald Rothschild (eds.) (1998), The international spread of ethnic conflict: fear, diffusion, and escalation, Princeton, NJ, Princeton University Press, 3-32 
-Lazzerini, Edward (1985), "Crimean tatar: the fate of a severed tongue", en: Kreindler, Isabelle T. (ed.) (1985), Sociolinguistic perspectives on soviet national languages: their past, present and future, Berlin, Mouton de Gruyter, pp. 109-124.

-Matsuo, Masatsugu (1999), "Language differentiation and homogenization in nested conflicts: two case studies", en: Journal of International Development and Cooperation, 5(1), pp. 87-102

-Matsuo, Masatsugu (2005), "One language or two? Real and perceived identification and differentiation of language", en: Hiroshima Peace Science, 27, pp. 189-203.

-Musa, Monsur (1996), "Politics of Language Planning in Pakistan and the Birth of a New State", en: International Journal of the Sociology of Language, 118, pp. 63-80.

-Nelde, Peter H. (1987), "Language contact means language conflict", en: Gearoid Mac Eoin et al (eds.), Third international conference on minority languages, General Papers, Clevedon, Multilingual Matters, pp. 33-42.

-Ozolins, Uldis (1996), "Language policy and political reality", en: International journal of the sociology of language, 118, pp. 181-200.

-Parkinson, Stephen (1990), "Portuguese", en: Comrie, Bernard (ed.) (1990a), The major languages of western europe, London, Routledge, pp. 250-268.

-Rothschild, Joseph (1981), Ethnopolitics: a conceptual framework, New York, Columbia Univesity Press.

-Safran, William (1999), "Nationalism”, en: Fishman, Joshua A. (ed.) (1999), Handbook of language and ethnic identity, New York: Oxford University Press, pp. 77-93.

-Schieffelin, Bambi B. and Rachelle Charlier Doucet (1998), “The 'real' haitian creole: ideology, metalinguisitics, and orthographic choice”, en: Schieffelin, Bambi B. (eds.), Language ideologies: practice and theory, New York, Oxford University Press, 285-316. Reprinted from American Ethnologist, 21(1) (1994). 
-Smith, Anthony. (1986), The ethnic origins of nations, Oxford, Basil Blackwell.

-Solchanyk, Roman (1985), "Language politics in the Ukraine", en: Kreindler, Isabelle T. (ed.) (1985), Sociolinguistic perspectives on soviet national languages: their past, present and future, Berlin, Mouton de Gruyter, pp. 57-105.

-Steinberg, Jonathan (1987), "The historian and the questione della lingua", en Burk, Peter, and Roy Porter (eds.), The social history of language, Cambridge, Cambridge University Press, pp. 198-209

-Subtelny, Orest (1988), Ukraine: a history, Toronto, University of Toronto Press.

-Weinstein, Brian (1989), "Francophonie: purism at the international level", Bjorn H. Jernudd and Michael J. Shapiro (eds.), The politics of language purism, Berlin, Mouton de Gruyter, pp. 53-79

-Wexler, Paul (1985), "Belorussification, russification and polonization trends in the belorussian language, 1890-1982”, en: Kreindler, Isabelle T. (ed.) (1985), Sociolinguistic perspectives on soviet national languages: theirpast, present and future, Berlin, Mouton de Gruyter, pp. 37-56. 